\title{
Time dependent multi-term solution of Boltzmann's equation for magnetised low temperature plasmas
}
R. D. White ${ }^{1}$
S. Dujko ${ }^{2}$
K. F. Ness ${ }^{3}$
R. E. Robson ${ }^{4}$
Z. Raspopović ${ }^{5}$
Z. Lj. Petrović ${ }^{6}$

(Received 25 August 2006; revised 27 April 2007)

\begin{abstract}
We present details of the first time dependent multi-term solution of Boltzmann's equation for charged particles in gases under the influence of electric and magnetic fields. A Sonine polynomial representation of the velocity distribution function requires a time dependent weight function to accommodate arbitrary initial conditions and/or time dependent fields. The theory and code are benchmarked against an independent time resolved Monte Carlo simulation for both DC and RF orthogonal electric and magnetic fields. The results support the numerical integrity of the technique.
\end{abstract}

See http://anziamj.austms.org.au/ojs/index.php/ANZIAMJ/article/view/97 for this article, (c) Austral. Mathematical Soc. 2007. Published May 1, 2007. ISSN $1446-8735$ 


\section{Contents}

1 Introduction

C70

2 Theory

C72

$\begin{array}{lll}3 & \text { Results and discussion } & \mathbf{C 7 7}\end{array}$

3.1 Transient relaxation . . . . . . . . . . . . . . C C77

3.2 Radiofrequency $E$ and $B$ fields . . . . . . . . . C C79

4 Conclusion

C80

References

C85

\section{Introduction}

Non-equilibrium, low temperature plasma discharges sustained by time dependent electric and magnetic fields are widely used in fabrication of microelectronic device and in the manufacturing of new materials. These plasmas are complex physical systems consisting of electrons, ions, molecules and radicals together with applied and space-charge fields. In general the electrons play the most important role, both in the discharge maintenance through electron-impact ionization, and in the production of radicals for chemical etching and ions for physical etching. Numerical models of these plasma discharges are critically dependent on an accurate knowledge of the electron kinetics in the collision dominated bulk of the plasma.

Accurate modelling of electron transport in gases under the influence of electric and magnetic fields is usually conducted through a solution of the appropriate Boltzmann equation solution for the velocity distribution function or through the use of Monte Carlo simulations. An approximate numerical technique for the solution of Boltzmann's equation, dominant in much of 
plasma physics, is referred to as the 'two-term' approximation (see the review by White et al. [13]). In this technique the velocity distribution function is assumed a priori to be quasi-isotropic and representable by only the first two terms of a Legendre polynomial basis set. The assumption greatly reduces the complexity involved in the numerical solution of Boltzmann's equation to the solution of a single second order differential equation. However, there are severe limitations on accuracy. Within the two-term framework there is no provision to increase the number of terms in the Legendre polynomial expansion and hence the accuracy of the technique has no provision for improvement. For accurate solutions of Boltzmann's equations, 'multi-term' techniques (see the review by Robson \& Ness [8]) are required where the number of terms in the expansion are incremented until convergence criteria are met. In general, the numerical schemes used in the two-term approximation do not carry over to multi-term techniques.

Recently, time dependent multi-term solutions of Boltzmann's equation for electric field only were independently developed by the groups at Griefswald [14, e.g.] and at JCU [12, e.g.], and more recently the group at Brno [9, e.g.]. The direct numerical procedure adopted by the Makabe group avoids a spherical harmonic expansion and is of comparable accuracy [2]. The development of these theories overcome various approximations used extensively within the field including the two-term approximation (which restricted gas types and electric field amplitudes) and the effective field approximation for oscillatory fields (which restricted field frequencies and/or gas densities). The extension to include electric and magnetic fields was subsequently made by the Griefswald group using a two-term Legendre polynomial expansion. The extension to a multi-term theory was made by the JCU group. The current theory and associated code adapts the mathematical and computational machinery developed for DC steady state swarms in $E$ and $B$ fields [3] to present the first time dependent multi-term solution of Boltzmann's equation for electric and magnetic fields (at arbitrary angles).

Boltzmann equation solutions and/or Monte Carlo simulations used in 
plasma discharge models should be benchmarked against known results under field conditions operative within the plasma discharge. The same applies for fluid models of electron transport, which effectively use low order moments of the Boltzmann equation, widely used in plasma discharge models. The fluid plasma components inherently have accuracy restrictions associated with these low order truncations. However, they have the potential to provide an accurate plasma model through the appropriate adoption of transport data. The accuracy of fluid models (and other plasma models) should be benchmarked against accurate techniques such as Boltzmann equation solutions and/or Monte Carlo simulations in the swarm limit; that is, in the limit of small electron densities the theories should reconcile each other. Recently, a benchmark model simulating field conditions in the bulk of an inductively coupled plasma was presented by the Petrović group using a Monte Carlo simulation [7]. The model unearthed a multitude of interesting phenomena for diffusion including the existence of transient negative diagonal diffusion elements. The focus of the present paper is to benchmark the current time dependent multi-term solution of Boltzmann's equation against this Monte Carlo technique for the model presented by Raspopović et al. [7]. Both techniques have their advantages/disadvantages. The increased mathematical complexity of Boltzmann equation solutions (as compared with MC simulations), facilitates solutions which that are typically at least one (but generally more) order of magnitude faster than MC simulations for the same accuracy.

\section{Theory}

The behaviour of a uniform swarm of electrons in gases under the influence of electric and magnetic fields is described by the Boltzmann equation. This equation represents the time $t$ evolution of the distribution function $f(\boldsymbol{c}, t)$ in velocity space $\boldsymbol{c}$. The distribution function is defined such that $f(\boldsymbol{c}, t) d \boldsymbol{c}$ is the probability of finding a particle within $d \boldsymbol{c}$ of $\boldsymbol{c}$ at time $t$. The explicit form of Boltzmann's equation for charged particle of charge $q$ and mass $m$ under 
the influence of spatially homogeneous orthogonal electric $\boldsymbol{E}$ and magnetic $\boldsymbol{B}$ fields is

$$
\frac{\partial f}{\partial t}+\frac{q}{m}[\boldsymbol{E}+\boldsymbol{c} \times \boldsymbol{B}] \cdot \frac{\partial f}{\partial \boldsymbol{c}}=-J\left(f, f_{0}\right) .
$$

Swarm conditions are assumed to apply and $J\left(f, f_{0}\right)$ denotes the rate of change of $f$ due to binary, particle conserving collisions with the neutral molecules only. The original Boltzmann collision operator and its semiclassical generalisation [10] are used for elastic and inelastic processes respectively:

$$
J\left(f, f_{0}\right)=\sum_{j k} \int\left[f(\boldsymbol{c}, t) f_{0 j}\left(\boldsymbol{c}_{0}\right)-f\left(\boldsymbol{c}^{\prime}, t\right) f_{0 k}\left(\boldsymbol{c}_{0}^{\prime}\right)\right] g \sigma(j k ; g \chi) d \hat{\boldsymbol{g}}^{\prime} d \boldsymbol{c}_{0}
$$

Dashed and undashed quantities refer to post- and pre-collision properties respectively. This form of the conservative collision operator treats internal states quantum mechanically and translational states classically. The quantity $\sigma(j k ; g \chi)$ is the differential cross-section describing the the scattering of a swarm particle of velocity $\boldsymbol{c}$, from a neutral molecule in the $j$ th internal state of velocity $\boldsymbol{c}_{0}$. The post-collision swarm particle and neutral velocities, and the final internal state of the neutral molecule are denoted by $\boldsymbol{c}^{\prime}, \boldsymbol{c}_{0}^{\prime}$ and $k$ respectively. The neutral molecules are assumed to remain in thermal equilibrium, characterized by a spatially homogeneous Maxwellian velocity distribution function $f_{0}\left(\boldsymbol{c}_{0}\right)$. The quantity $d \hat{\boldsymbol{g}}^{\prime}=\sin \chi d \chi d \zeta$ represents the elements of angles of the post-collision relative velocity where $\chi$ and $\zeta$ are the scattering angles. In what follows, we employ a co-ordinate system in which $\boldsymbol{E}$ is in the $z$-direction, while $\boldsymbol{B}$ is in the $y$-direction.

Solution of (1) has been detailed by White et al. [12] and we present here only a brief outline. The following representations of the phase space distribution function $f$ are made under spatially homogeneous conditions. 
Resolution of the angular dependence in velocity space The angular dependence is represented in terms of a spherical harmonic expansion,

$$
f(\boldsymbol{c}, t)=\sum_{l=0}^{\infty} \sum_{m=-l}^{l} f_{m}^{(l)}(c, t) Y_{m}^{[l]}(\hat{\boldsymbol{c}}),
$$

where $Y_{m}^{[l]}(\hat{\boldsymbol{c}})$ are spherical harmonics which are orthonormal on the angles of $\boldsymbol{c}, \hat{\boldsymbol{c}}$. The superscripts [] and () represent standard and contrastandard spherical tensor forms respectively [1].

Resolution of the speed dependence The speed distribution function is represented by an expansion about a Maxwellian at a temperature $T_{b}$ in terms of modified Sonine polynomials:

$$
f_{m}^{(l)}(c, t)=w(\alpha, c) \sum_{\nu=0}^{\infty} F_{m}^{(\nu l)}(\alpha, t) R_{\nu l}(\alpha c),
$$

where

$$
\begin{aligned}
& w(\alpha, c)=\left(\frac{\alpha^{2}}{2 \pi}\right)^{3 / 2} \exp \left\{\frac{-\alpha^{2} c^{2}}{2}\right\}, \\
& R_{\nu l}(\alpha c)=N_{\nu l}\left(\frac{\alpha c}{\sqrt{2}}\right)^{l} S_{l+1 / 2}^{(\nu)}\left(\frac{\alpha^{2} c^{2}}{2}\right), \\
& N_{\nu l}^{2}=\frac{2 \pi^{3 / 2} \nu !}{\Gamma(\nu+l+3 / 2)},
\end{aligned}
$$

$S_{l+1 / 2}^{(\nu)}\left(\frac{1}{2} \alpha^{2} c^{2}\right)$ are Sonine polynomials, and $\alpha^{2}=m /\left(k T_{b}\right)$. The modified Sonine polynomials satisfy the orthonormality relation

$$
\int_{0}^{\infty} w(\alpha, c) R_{\nu^{\prime} l}(\alpha c) R_{\nu l}(\alpha c) c^{2} d c=\delta_{\nu^{\prime} \nu}
$$


Ness and White [5] further comment on the use of Sonine polynomials in kinetic theory.

Making use of the orthogonality properties of the basis functions, the following complex doubly infinite coupled differential equations are generated under conservative conditions [12]:

$$
\begin{aligned}
\sum_{\nu^{\prime}=0}^{\infty} \sum_{l^{\prime}=0}^{\infty} \sum_{m^{\prime}=-l^{\prime}}^{l^{\prime}}[ & \left(N \frac{d}{d t} \delta_{\nu \nu^{\prime}}+N J_{\nu \nu^{\prime}}^{l}(\alpha)\right) \delta_{l^{\prime} l} \delta_{m^{\prime} m} \\
& +i \frac{q E}{m} \alpha\left(l^{\prime} m 10 \mid l m\right)\left\langle\nu l\left\|K^{[1]}(\alpha)\right\| \nu^{\prime} l^{\prime}\right\rangle \delta_{m^{\prime} m} \\
& +\frac{1}{2} \frac{q B}{m}\left\{\sqrt{(l-m)(l+m+1)} \delta_{m^{\prime} m+1}\right. \\
& \left.\left.-\sqrt{(l+m)(l-m+1)} \delta_{m^{\prime} m-1}\right\} \delta_{l^{\prime} l} \delta_{\nu^{\prime} \nu}\right] F_{m^{\prime}}^{\left(\nu^{\prime} l^{\prime}\right)}=0
\end{aligned}
$$

where $N$ is the neutral number density and $\left(l^{\prime} m 10 \mid l m\right)$ is a Clebsch-Gordan coefficient [1]. The explicit expressions for the reduced matrix elements are given by White et al. [12]. Discretising in time using an implicit finite difference scheme converts the system of coupled differential equations into a system of coupled matrix equations. We note $\nu=0, \ldots, \infty, l=0, \ldots, \infty$ and $m=0, \ldots, l$. Solution of (9) is made by truncation of the $\nu$ and $l$ indices to $\nu_{\max }$ and $l_{\max }$ respectively. These values are independently increased until the desired convergence on the calculated transport properties of interest is obtained. $T_{b}$ is a parameter used to optimise convergence of the speed space expansion and hence the value of $\nu_{\max }$ is coupled to the value of $T_{b}$. From previous experience with steady state calculations, a given $T_{b}$ can only give converged results over a restricted range of applied fields (or equivalently mean energies). Hence, it is essential that the parameter is time dependent to ensure convergence under time dependent conditions. Solution of the system of couple matrix equations is made through direct numerical inversion using a sparse matrix inversion routine [15]. There is some non-standard block structure in the equations but this has not been exploited. 
For the crossed field configuration, symmetry requirements dictate that the drift velocity vector can only have components in the $\boldsymbol{E}$ and $\boldsymbol{E} \times \boldsymbol{B}$ directions. The drift velocity components and the mean energy are expressed directly in terms of the calculated moment:

$$
\begin{aligned}
& W_{E \times B}=\frac{1}{\alpha} \sqrt{2} \Im\left\{F_{1}^{(01)}\right\}, \\
& W_{E}=-\frac{1}{\alpha} \Im\left\{F_{0}^{(01)}\right\}, \\
& \varepsilon=\frac{3}{2} k T_{b}\left[1-\sqrt{\frac{2}{3}} \Re\left\{F_{0}^{(10)}\right\}\right],
\end{aligned}
$$

where $\Re\{\}$ and $\Im\{\}$ respectively represent the real and imaginary parts of the moments.

For the crossed field configuration, the following symmetry property exists in the moments,

$$
F_{-m}^{(\nu l)}=(-1)^{m} F_{m}^{(\nu l)}
$$

whereas the reality of the distribution function implies

$$
\left(F_{-m}^{(\nu l)}\right)^{*}=(-1)^{l+m} F_{m}^{(\nu l)} .
$$

On combining these relations we have

$$
\left(F_{m}^{(\nu l)}\right)^{*}=(-1)^{l} F_{m}^{(\nu l)},
$$

and it follows that the system of complex equations can be recast into a form where the renormalised moments are real and only non-negative values of $m$ are required. This greatly reduces the computational time.

This concludes our brief description of the theoretical and computational formalism. In the next section, we apply this theory to the multi-term calculation of temporal relaxation of transport coefficients for electron swarms under the action of electric and magnetic fields. 
TABLE 1: Benchmark calculations for steady state crossed DC electric and magnetic fields in the Reid ramp model. The current Boltzmann equation solution (Boltz) is compared to two independent Monte Carlo simulation techniques MC1 [11] and MC2 [6].

\begin{tabular}{ccccc}
\hline $\begin{array}{c}\text { Transport } \\
\text { property }\end{array}$ & $\begin{array}{c}B / N \\
(\mathrm{Hx})\end{array}$ & Boltz & $\begin{array}{c}\mathrm{MC} 1 \\
{[11]}\end{array}$ & $\begin{array}{c}\mathrm{MC} 2 \\
{[6]}\end{array}$ \\
\hline$\varepsilon(\mathrm{eV})$ & 0 & 0.2689 & 0.2693 & 0.2703 \\
$W_{E}\left(10^{4} \mathrm{~ms}^{-1}\right)$ & & 6.838 & 6.833 & 6.834 \\
$\varepsilon(\mathrm{eV})$ & 500 & 0.1123 & 0.1124 & 0.1133 \\
$W_{E}\left(10^{4} \mathrm{~ms}^{-1}\right)$ & & 2.318 & 2.318 & 2.3180 \\
$W_{E \times B}\left(10^{4} \mathrm{~ms}^{-1}\right)$ & & 0.4154 & 0.4161 & 0.4170 \\
\hline
\end{tabular}

\section{Results and discussion}

\subsection{Transient relaxation}

The initial benchmark of the time dependent theory and associated code is the transient response to a superimposed magnetic field and its asymptotic solution using the Reid ramp model $[4,11,7,6]$. For $t<0$ the swarm is acted on solely by a DC electric field $(E / N=12 \mathrm{Td}, B / N=0 \mathrm{Hx}$; $\left.1 \mathrm{Td}=10^{-21} \mathrm{~V} \mathrm{~m}^{2}, 1 \mathrm{Hx}=10^{-27} \mathrm{~T} \mathrm{~m}^{3}\right)$ is allowed to relax to a steady state. At time $t=0$, a crossed magnetic field is switched on (electric field is unaltered) and the relaxation properties of the swarm are monitored as a function of time. The temporal variation of the results are a function of $N t$. An example of the influence of the magnetic field on the transient response calculated using the multi-term solution of Boltzmann's equation is displayed in Figure 1. The asymptotic results are displayed in Table 1 where they are compared with two independent Monte Carlo simulation procedures. Over the range of conditions and transport properties considered the current theory has agreement with the MC techniques to better than $1 \%$ and support the numerical integrity of the theory and code. 


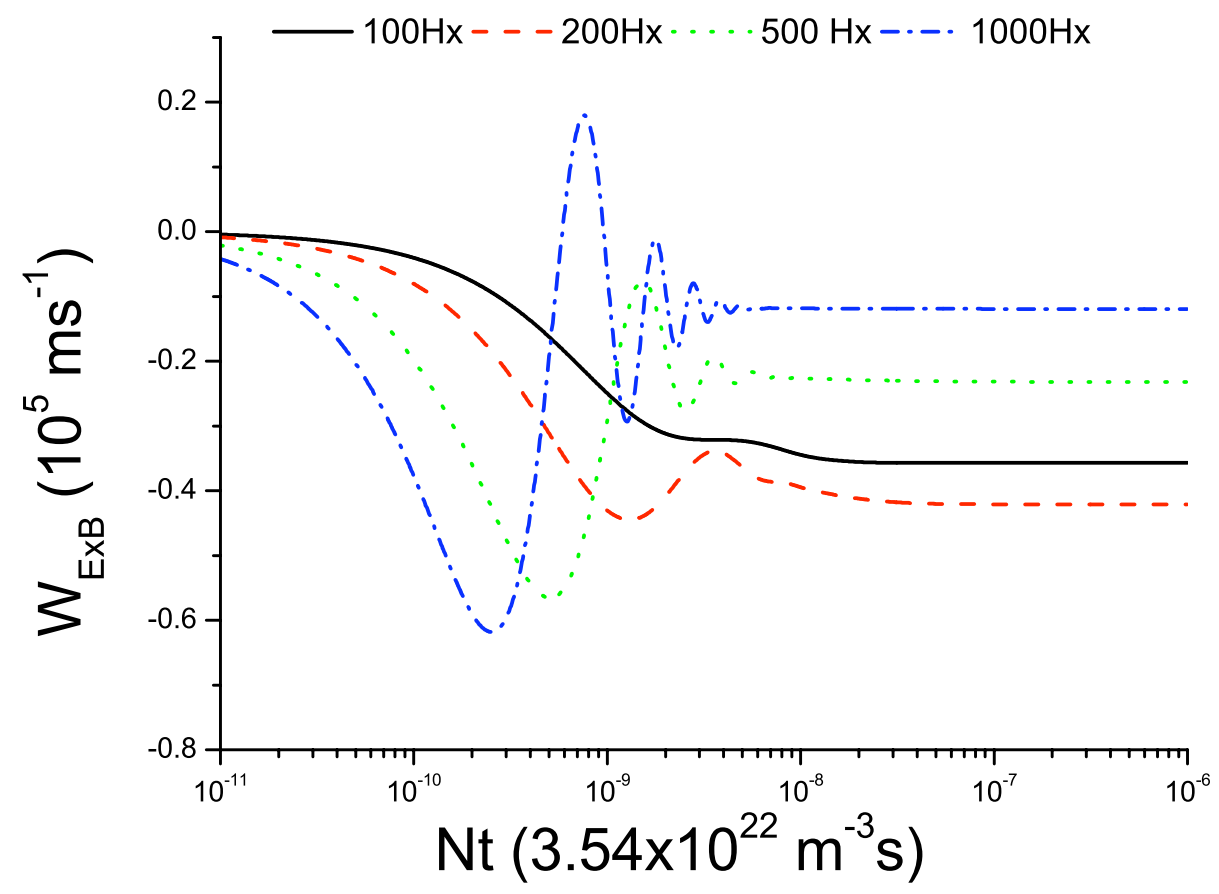

FigURE 1: Multi-term solution demonstrating the influence of magnetic field strength on the transient response of $W_{E \times B}$ to the application of an orthogonal magnetic field. 
TABLE 2: Convergence in the $l$-index for various transport coefficients and properties as a function of $B / N .(E / N=12 \mathrm{Td})$

\begin{tabular}{cccccccc}
\hline $\begin{array}{c}\text { Transport } \\
\text { property }\end{array}$ & $\begin{array}{c}B / N \\
(\mathrm{Hx})\end{array}$ & 1 & 2 & 3 & 4 & 5 & 6 \\
\hline$\varepsilon$ & 0 & 0.2736 & 0.2689 & 0.2689 & 0.2690 & 0.2689 & 0.2689 \\
$(\mathrm{eV})$ & 500 & 0.1121 & 0.1123 & 0.1123 & 0.1123 & 0.1123 & 0.1123 \\
$W_{E \times B}$ & 0 & 0 & 0 & 0 & 0 & 0 & 0 \\
$\left(10^{4} \mathrm{~ms}^{-1}\right)$ & 500 & 2.317 & 2.318 & 2.318 & 2.318 & 2.318 & 2.318 \\
$W_{E}$ & 0 & 7.030 & 6.821 & 6.841 & 6.837 & 6.839 & 6.838 \\
$\left(10^{4} \mathrm{~ms}^{-1}\right)$ & 500 & 0.4156 & 0.4154 & 0.4154 & 0.4154 & 0.4154 & 0.4154 \\
\hline
\end{tabular}

It is interesting to study the convergence of the asymptotic results in the $l$-index. The results are displayed in Table 2 . For $B / N=0$ there exists a well known anisotropy in the velocity distribution function [4]. Hence, we observe that an $l_{\max }$ of $4-5$ is required to achieve convergence to within $0.1 \%$. When a strong magnetic field of $500 \mathrm{Hx}$ is applied however we observe a significant reduction in the anisotropy of the velocity distribution. This is due to the action of the Lorentz force causing the electrons to orbit with a (cyclotron) period that is less than the mean time between collisions.

\subsection{Radiofrequency $E$ and $B$ fields}

The second benchmark involved oscillatory $E$ and $B$ fields. The current technique and code was benchmarked against an independent time resolved Monte Carlo simulation technique developed at Belgrade University [6] for the Reid ramp model for the conditions detailed by Raspopović et al. [7]. The RF fields are orthogonal, $\pi / 2$ out of phase and spatially homogeneous $(E / N=10 \sqrt{2} \sin (2 \pi f t) \mathrm{Td}, B / N=500 \cos (2 \pi f t) \mathrm{Hx}$, where $f$ is the field frequency). The neutral gas density is fixed at $N=3.54 \times 10^{22} \mathrm{~m}^{-3}$. The results are displayed in Figures 2-4 for the transport coefficients of interest 
where they are compared to the Monte Carlo simulation technique. We tested the theory and code over a range of conditions for which the traditional approximations and assumptions fail. These comparative results support the accuracy and integrity of the current theory and associated code. We finish with a few comments on the generality of the current theory and implications on certain results.

The temporal profiles of the mean energy and drift velocity components are in general not predictable from steady state DC results. Perhaps the best example of this the presence of high frequency oscillations (higher than the field frequency) in both the $W_{E}$ and $W_{E \times B}$ profiles as the field frequency is increased. These high frequency oscillations represent essentially collisionless gyro-orbits of the swarm particles. Hence, plasma models that utilise and extrapolate DC data only should quantify the errors associated with such an approximation. The need for a genuine multi-term theory is well known since the anisotropy of the velocity distribution function is in general not know a priori. Figure 5 compares the two-term and converged multi-term solutions. We see that at those phases where $B / N$ is small, we have an increased distinction between the two-term and multi-term profiles. The phase where this distinction is a maximum is delayed as the frequency is increased. When the magnitude of the instantaneous values of $B / N$ are at or near the maximum, the two-term approximation becomes sufficient. Phenomenologically, the two-term approximation produces the correct results for this particular model, though this is not always the case.

\section{Conclusion}

In this work we presented the details of the first systematic time dependent multi-term solution of Boltzmann's equation for electrons in gases under the influence of electric and magnetic fields. The technique was applied to a series of benchmark models involving DC and RF electric and magnetic 


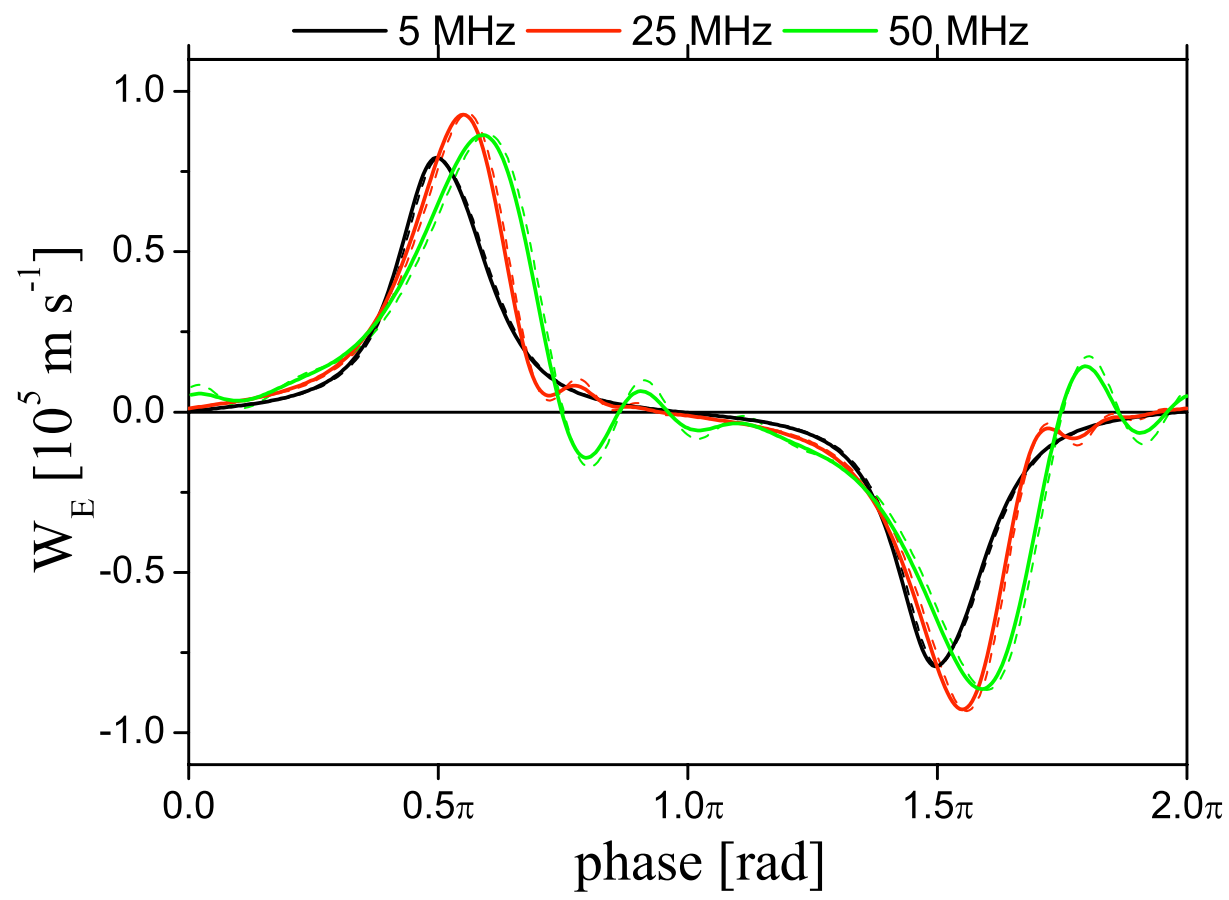

Figure 2: Comparison of the Boltzmann (solid) and Monte Carlo (dashed) results for $W_{E}$ over a range of applied frequencies for the Reid ramp model. 


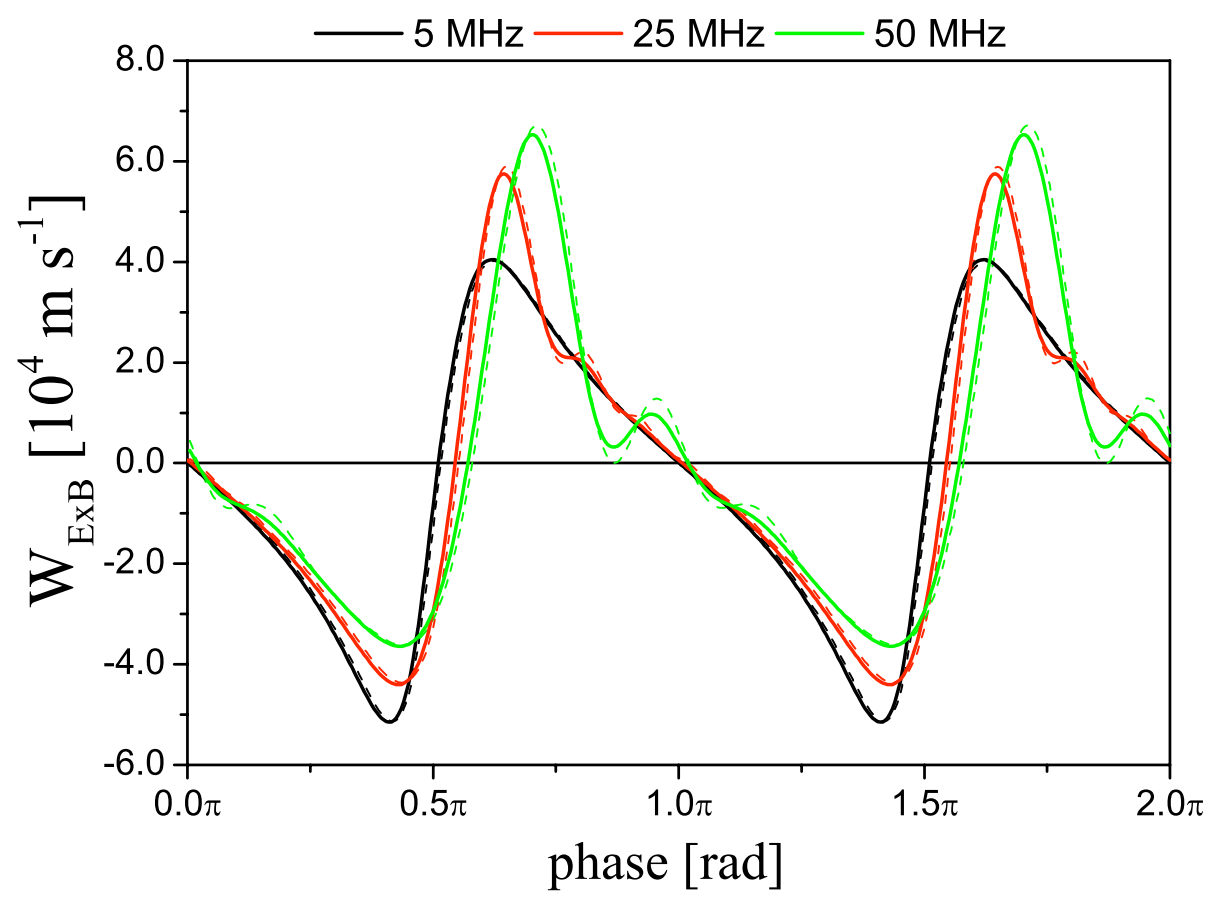

Figure 3: Comparison of the Boltzmann (solid) and Monte Carlo (dashed) results for $W_{E \times B}$ over a range of applied frequencies for the Reid ramp model. 


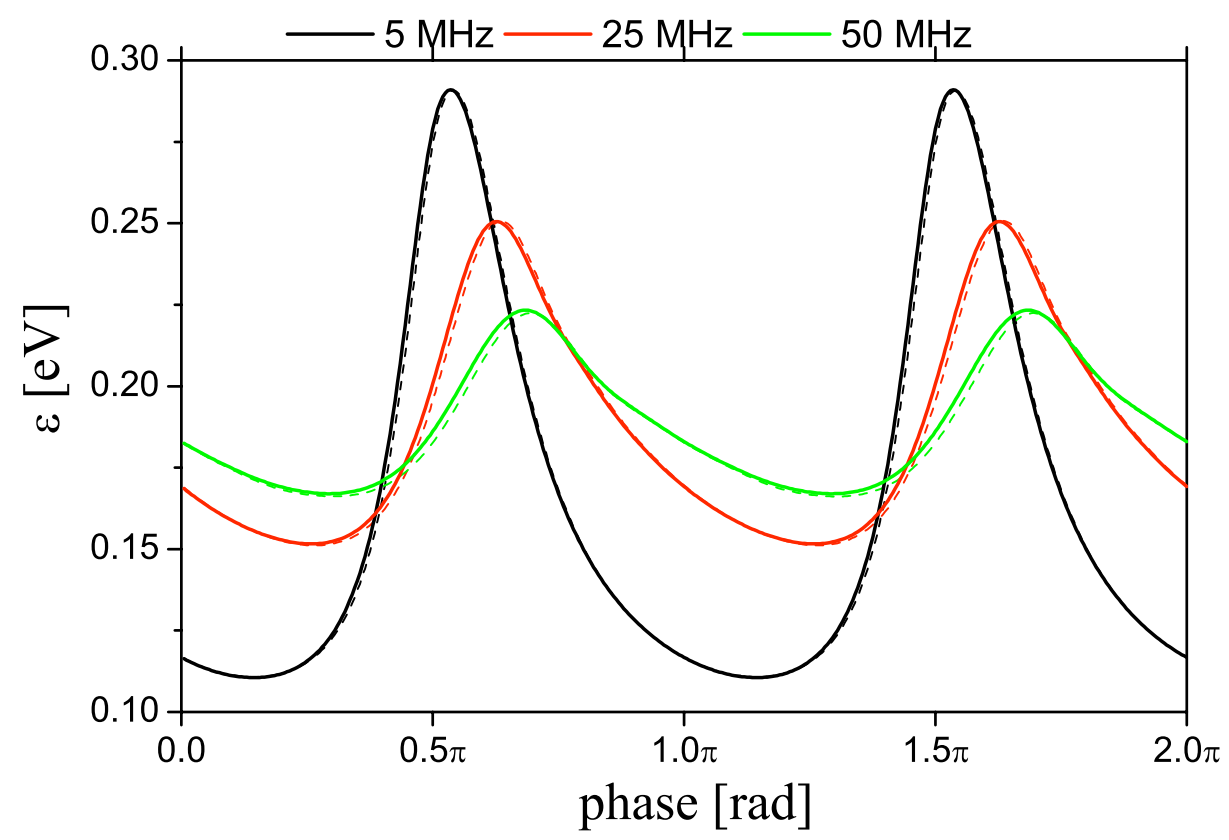

Figure 4: Comparison of the Boltzmann (solid) and Monte Carlo (dashed) results for $\varepsilon$ over a range of applied frequencies for the Reid ramp model. 

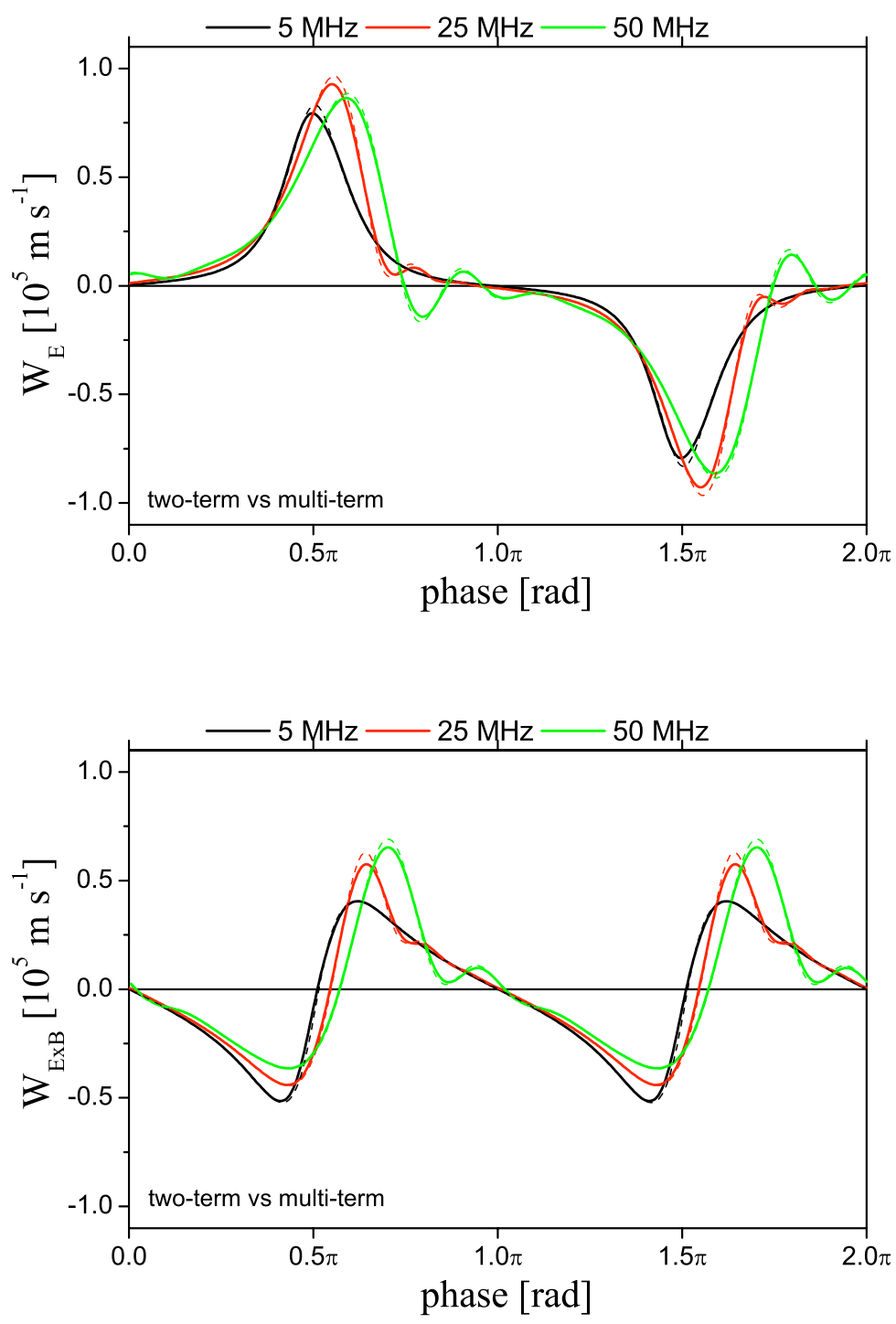

Figure 5: Comparison of the two-term (dashed) and converged multi-term (solid) Boltzmann results for $W_{E}$ and $W_{E \times B}$ over a range of applied frequencies for the Reid ramp model. 
fields and compared with those from a time resolved Monte Carlo simulation developed at the University of Belgrade. The results support the integrity of the present technique and code and the need for a genuine multi-term technique was illustrated.

\section{References}

[1] U. Fano and G. Racah, Irreducible tensorial sets (Academic, New York, 1959). C74, C75

[2] K. Maeda, T. Makabe, N. Nakano, S. Bzenić and Z. Lj. Petrović, "Diffusion tensor in electron transport in gases in radio-frequency field", Phys. Rev. E 55 (1997) 5901. doi:10.1103/PhysRevE.55.5901 $\mathrm{C} 71$

[3] K. F. Ness, "Multi-term solution of the Boltzmann equation for electron swarms in crossed electric and magnetic fields", J. Phys. D: Appl. Phys. 27 (1994) 1848. doi:10.1088/0022-3727/27/9/007 C71

[4] K. F. Ness and R. E. Robson, "Velocity distribution function and transport coefficients of electrons in gases: Spherical harmonic decomposition of the Boltzmann equation", Phys. Rev. A 34 (1986) 2185. doi:10.1103/PhysRevA.34.2185 C77, C79

[5] K. F. Ness and R. D. White, "Burnett function expansions with a bi-Maxwellian weight function for electron swarm physics" ANZIAM Journal, to appear. http://anziamj.austms.org.au/ojs/index. php/ANZIAMJ/article/view/123 C75

[6] Z. Lj. Petrović, Z. M. Raspopović, S. Dujko and T. Makabe, "Kinetic phenomena in electron transport in radio frequency fields", Appl. Surf. Sci. 192 (2002) 1-21. doi:10.1016/S0169-4332(02)00018-1 C77, C79 
[7] Z. Raspopović, S. Sakadžić, Z. Lj. Petrović and T. Makabe, "Diffusion of electrons in time-dependent $e(t) \times b(t)$ fields", J. Phys. D: Appl. Phys. (2000) 1298.. doi:10.1088/0022-3727/33/11/308 C72, C77, C79

[8] R. E. Robson and K. F. Ness, "Velocity distribution function and transport coefficients of electrons in gases 2: Moment equations and applications", Phys. Rev. A 33 (3) (1986) 2068. doi:10.1103/PhysRevA.33.2068 C71

[9] D. Trunec, Z. Bonaventura and D. Necas, "Solution of the time-dependent boltzmann equation for electrons in non-thermal plasma", J. Phys. D: Appl. Phys. 39 (2006) 2544-2552. doi:10.1088/0022-3727/39/12/012 C71

[10] C. S. Wang-Chang, G. E. Uhlenbeck and J. De Boer, in Studies in Statistical Mechanics (eds. J. De Boer and G. E. Uhlenbeck), Volume II, (Wiley, New York, 1964), 241. C73

[11] R. D. White, M. J. Brennan and K. F. Ness, "Benchmark simulations for electron swarms in crossed electric and magnetic fields", J. Phys. D: Appl. Phys. 30 (1997) 810-816. doi:10.1088/0022-3727/30/5/014 C77

[12] R. D. White, K. F. Ness and R. E. Robson, "Development of swarm transport theory in radio-frequency electric and crossed electric and magnetic fields", Appl. Surf. Sci. 192 (2002) 26-49. doi:10.1016/S0169-4332(02)00019-3 C71, C73, C75

[13] R. D. White, R. E. Robson, B Schmidt and M. A. Morrison, "Is the classical two-term approximation of electron kinetic theory satisfactory for swarms and plasmas?", J. Phys. D: Appl. Phys. 36 (2002) 3125-3131. doi:10.1088/0022-3727/36/24/006 C71

[14] R. Winkler, D. Loffhagen and F. Sigeneger, "Temporal and spatial relaxation of electrons in low temperature plasmas", Applied Suface Science 192 (2002) 50-71. doi:10.1016/S0169-4332(02)00020-X C71 
[15] Z. Zlatev, J. Wasniewski and K. Schaumburg, Y12M - Solution of Large and Sparse Systems of Linear Algebraic Equations (Springer, Berlin, 1981). C75 


\section{Author addresses}

1. R. D. White, School of Mathematics, Physics \& Information Technology, James Cook University, Townsville, Australia. mailto:Ronald. White@jcu.edu.au

2. S. Dujko, School of Mathematics, Physics \& Information Technology, James Cook University, Townsville, Australia.

3. K. F. Ness, School of Mathematics, Physics \& Information Technology, James Cook University, Townsville, Australia.

4. R. E. Robson, School of Mathematics, Physics \& Information Technology, James Cook University, Townsville, Australia.

5. Z. Raspopović, Institute of Physics, University of Belgrade, 11080 Zemun, SERBIA.

6. Z. Lj. Petrović, Institute of Physics, University of Belgrade, 11080 Zemun, SERBIA. 